\title{
A new 3D viewer as an interface between the ASDEX Upgrade CAD models and data from plasma modelling and experiment
} \author{
team $^{a}$ \\ ${ }^{a}$ Max-Planck-Institut für Plasmaphysik, Boltzmannstr. 2, D-85748 Garching \\ ${ }^{b}$ Max-Planck-Institut für Plasmaphysik, Wendelsteinstr. 1, D-17491 Greifswald \\ ${ }^{c}$ Institut Jean Lamour, Faculté des sciences, 54506 Vandoeuvre-lès-Nancy, FRANCE
}

T. Lunt ${ }^{a 1}$, J.C. Fuchs ${ }^{a}$, K. Mank ${ }^{a}$,Y. Feng ${ }^{b}$, F. Brochard ${ }^{c}$, A. Herrmann ${ }^{a}$, V. Rohde ${ }^{a}$, N. Endstrasser ${ }^{a}$ and the ASDEX Upgrade

\begin{abstract}
A generally available and easy-to-use viewer for the simultaneous visualisation of the ASDEX Upgrade vacuum vessel computer aided design models, diagnostics and magnetic geometry, solutions of 3D plasma simulation codes and 2D camera images was developed. Here we report on the working principle of this software and give several examples of its technical and scientific application.
\end{abstract}

Keywords: tomography, hot spot detection, virtual diagnostics

\section{Introduction}

The ASDEX Upgrade (AUG) vessel geometry, including diagnostic front ends is described to a high level of detail in the construction drawings created by the computer aided design (CAD) system MEDUSA. The handling of these complex 3DCAD models, however, is very time consuming and requires access to the drawing database and knowledge of its structure and interlinks. In practice this limits its usability as a visualisation tool. Since more and more 2D imaging diagnostics as well as modelling codes require 3D data visualisation a general and easy-to-use interface between the 3D-CAD model and external applications is highly desirable. In the following we will report on the working principle of such a viewer recently developed at AUG and give various examples for applications.

\section{Working principle}

Since triangles are the most elementary $2 \mathrm{D}$ object, any $2 \mathrm{D}$ surface in 3D space can be 'triangulated'. For this reason the majority of today's graphics cards are optimised to triangles. The 'Standard Triangulation Language', sometimes also called 'Surface Tessellation Language', (STL) is a format to store 3D geometry as a list of triangles. Being extremely simple, almost any CAD software supports this format. Since no further time consuming triangulation has to be performed the rendering of this data is very efficient. The high rendering speed, however, is at the expense of data volume, which can be much smaller in the case of other formats.

Augddd is a software tool based on openGL (1) technology to display STL files together with other geometry data developed at ASDEX Upgrade (AUG). The entire AUG vacuum vessel is exported from the CAD construction drawings as a data set consisting of as many as 20,000,000 triangles, occupying about 1
GB of binary data (for the standard user version this data set is reduced to about $200 \mathrm{MB}$ ). This data set is rendered by modern graphics cards within only a few seconds. However, in order to perform rotations and translations in real time the list of triangles is ordered by size, so that the user can reduce the level of detail depending on the individual application and the computing performance available.

Besides the STL files the user can display self defined 3D objects. Lines of sight (LOS) from spectroscopy and other diagnostics geometry information can be requested from diaggeom, a diagnostic geometry server at AUG, via the http protocol. An important feature of the software is that the geometry can be displayed from any virtual eye position defined by $\vec{e}$ (the vector pointing to the eye), $\vec{c}$ (a vector to a point on the central viewing chord) and $\vec{u}$ (a vector pointing upward). This is achieved by applying the 'camera' $4 \times 4$ transformation matrix

$$
C=\left(\begin{array}{cccc}
s_{x} & s_{y} & s_{z} & -\vec{s} \cdot \vec{e} \\
v_{x} & v_{y} & v_{z} & -\vec{v} \cdot \vec{e} \\
-f_{x} & -f_{y} & -f_{z} & \vec{f} \cdot \vec{e} \\
0 & 0 & 0 & 1
\end{array}\right),
$$

with $\vec{f}=\|\vec{c}-\vec{e}\|, \vec{s}=\|\vec{f} \times \vec{u}\|$ and $\vec{v}=\|\vec{s} \times \vec{f}\|$ to the $4 \mathrm{D}$ vector $(x, y, z, 1)^{T}$ corresponding to a point $(x, y, z)^{T}$ in $3 \mathrm{D}$ space. Working in the 4D instead of the 3D space translations and rotations can be stored in the same transformation matrix. The reader may assure oneself immediately that $C \cdot(\vec{e}, 1)^{T}=(0,0,0,1)^{T}, C \cdot(\vec{e}+\vec{f}, 1)^{T}=(0,0,-1,1)^{T}$ and $C \cdot(\vec{e}+\vec{v}, 1)^{T}=(0,1,0,1)^{T}$. After applying the camera transformation a line $\vec{e}+\lambda \vec{f}, \lambda=0 \ldots \infty$ thus appears as a point. Fig. 1 shows a virtual view into the AUG vacuum vessel. AUG is divided into 16 sectors, which are indicated by the red numbers. Since a tokamak to a high degree is toroidally symmetric anything plotted in 3D can also be shown in poloidal 


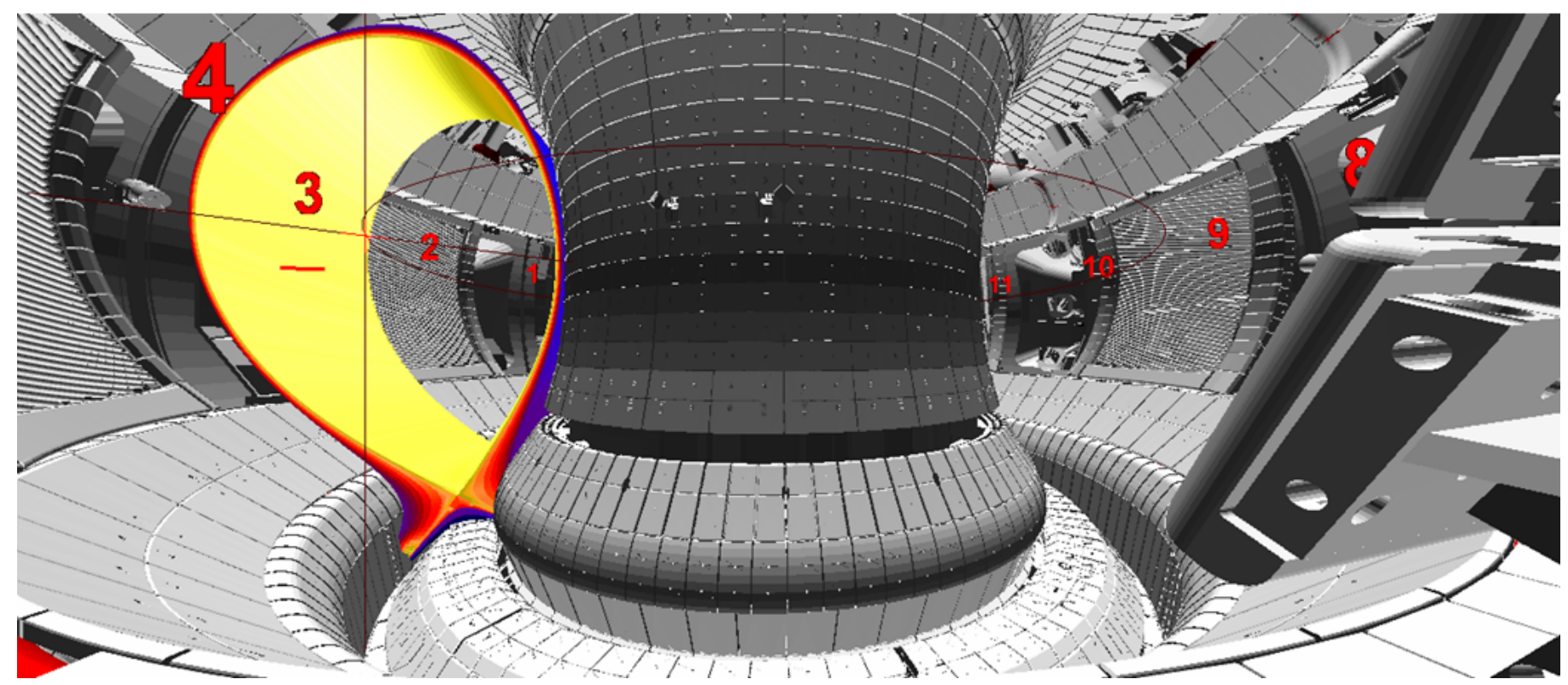

Figure 1: Virtual view into the AUG vacuum vessel, displayed together with a density distribution computed by EMC3-Eirene

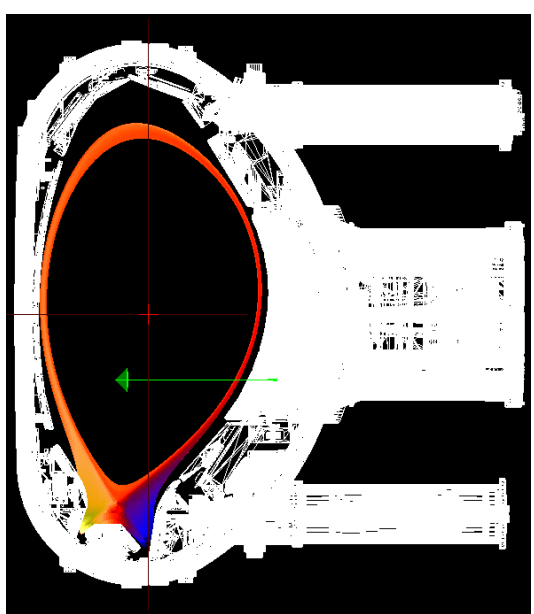

Figure 2: Poloidal projection of the geometry displayed in Fig. 1. The green arrow indicates the line of sight in the $3 \mathrm{D}$ mode.

projection (cf. Fig. 2) The 3D geometry can be compared to $2 \mathrm{D}$ camera images by projecting the image onto a $2 \mathrm{D}$ mesh $\vec{m}_{i, j}=\left[\left(m_{x}, m_{y}\right)^{T}\right]_{i, j}$ on a semi-transparent plane

$$
\vec{p}\left(m_{x}, m_{y}\right)=\vec{e}+c \vec{f}+m_{x} \vec{s}+m_{y} \vec{v}, c=\text { const. }
$$

plotted into the same openGL space. Different transformations, like translations, rotations but also barrel distortions (to correct the fish eye effect of short focal length optics) can be applied to the grid points $\vec{m}_{i, j}$ in order to achieve a good matching between the image and the 3D geometry. The LOS through any mesh vertex are then given by $\vec{L}=\vec{e}+\lambda(\vec{p}-\vec{e})$. Using interpolation techniques the LOS through every pixel on the image can be determined. This technique allows an accurate spatial calibration of the viewing geometry, hardly possible with mechanical techniques inside the vacuum vessel.

\section{Applications}

This accurate calibration is of high practical as well as scientific importance. It allows, for example, the accurate localisation of hot spots on plasma facing components during experimental operation and thus a better control of the operational parameters. Fig. 3 shows a virtual camera view into the AUG vacuum vessel generated from the CAD construction drawings. It is overplotted by a CCD camera image recording the plasma discharge \#24792 over large parts of the visible spectrum $\left(\lambda_{H_{\alpha}}=656 \mathrm{~nm}\right.$ is blocked by a filter). Since the boundary between the hot core plasma and the colder scrape-off-layer, the so called separatrix was too far outside during this discharge the heat flux onto the tiles was extraordinary high provoking several hot spots at the edge of the tiles. Recognizing and localizing these events enables the operator to correct the separatrix position.

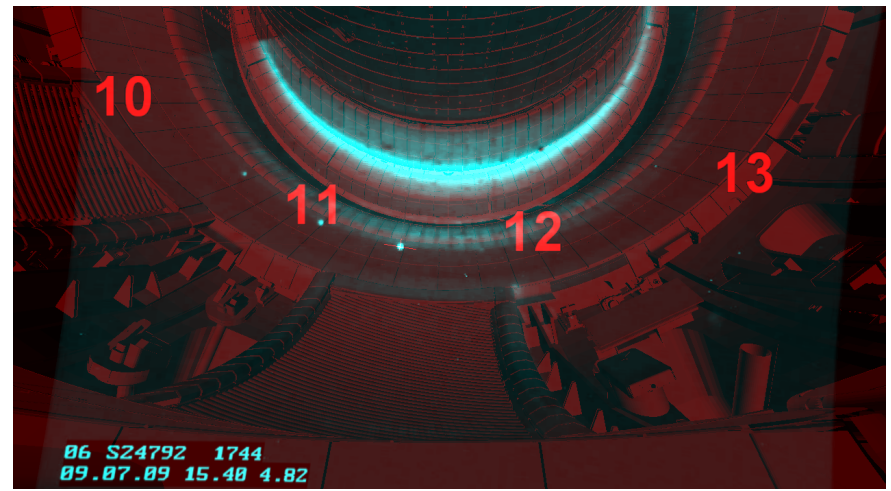

Figure 3: Virtual view into the AUG divertor (red) overplotted by 2D camera image (blue). The hot spots on the divertor tiles can be localized wit the help of the cursor (red cross).

The calibration is also essential when performing tomography. 
To a high degree a tokamak has a toroidal symmetry. With this assumption 3D radiation emission pattern of the plasma or the neutral particles can be deduced from a 2D camera image. This technique was applied by Fuchs and Gafert for the computation of the poloidal $465 \mathrm{~nm}$ (C III) emissivity profile in $(2 ; 3)$ and is important for impurity transport studies. The tomography algorithm can only work properly knowing the exact LOS corresponding to every pixel in the camera image.

Certain plasma volumes in AUG are observed simultaneously by two cameras from different viewing angles (cf. Fig. 4). If a strongly emitting object, like a pellet or a dust particle is observed by these two cameras, its $3 \mathrm{D}$ position can be determined finding the point of intersection (or at least the point of closest approximation) of the two LOS found by the cameras. Furthermore, if the time resolution of the cameras is high enough the 3D trajectory and thus (given that its mass is known) the time dependence of total force acting on the object can be reconstructed.
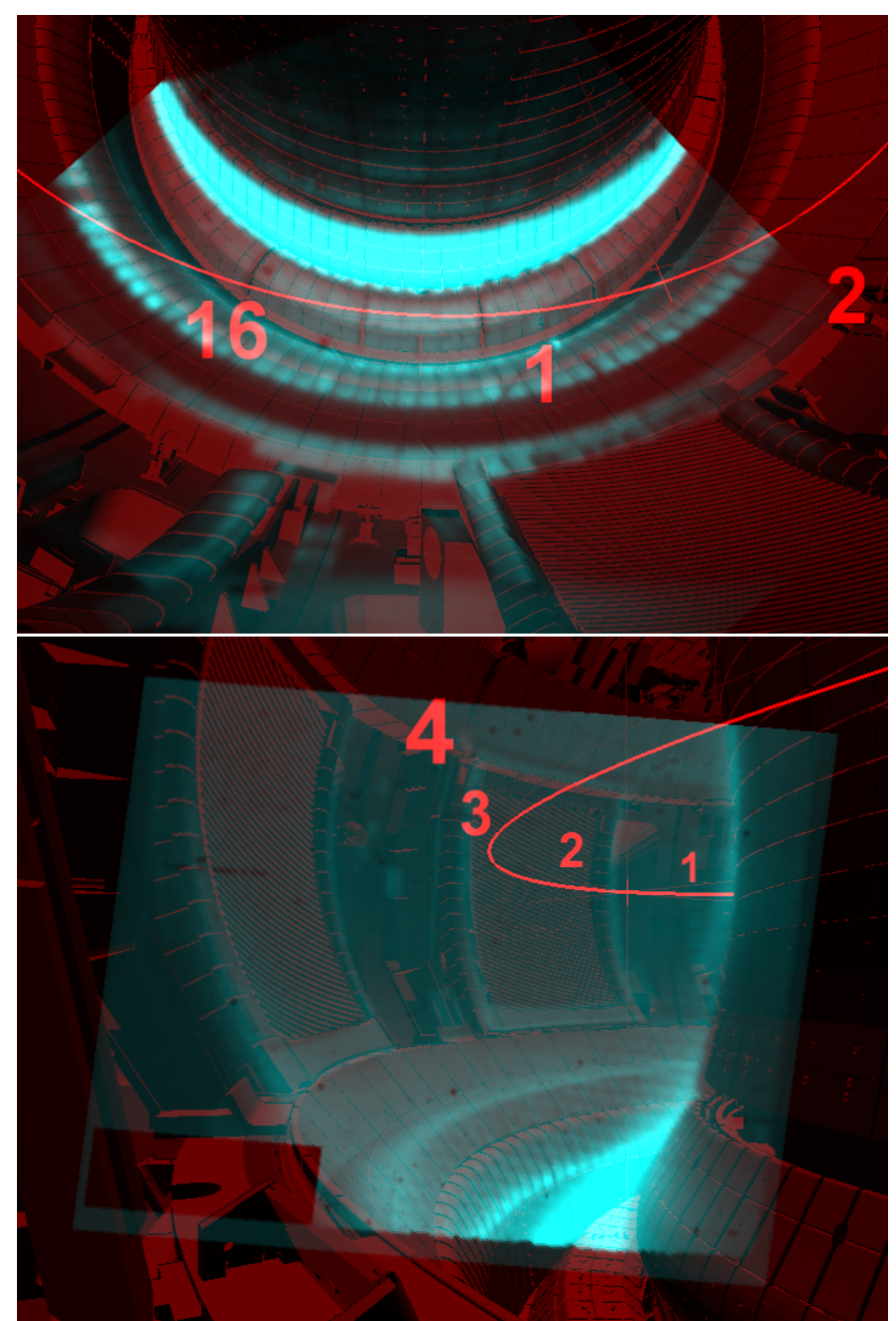

Figure 4: Two virtual views into the AUG vacuum vessel overplotted by 2D camera images observing the same plasma volume. Tracing a strongly emitting localized object, like a pellet or dust particle, its 3D trajectory can be reconstructed. The red lines indicates the field line passing the point $(\mathrm{R}, \mathrm{z}, \mathrm{phi})=\left(1.6 \mathrm{~m}, 0 \mathrm{~m}, 100^{\circ}\right)$.
The viewer can also be used to optimize the viewing geometry of new diagnostics and to detect conflicts between new invessel components and existing diagnostics in an early phase of component implementation. For example, this technique was applied during the design of the ELM control coils in AUG. In Fig. 5 a set of LOS of one of the AUG bolometers is shown. While all the LOS (red and green) could observe the interior of the vaccum vessel, the red lines will be blocked by the housing of the ELM control coils to be installed in 2010.

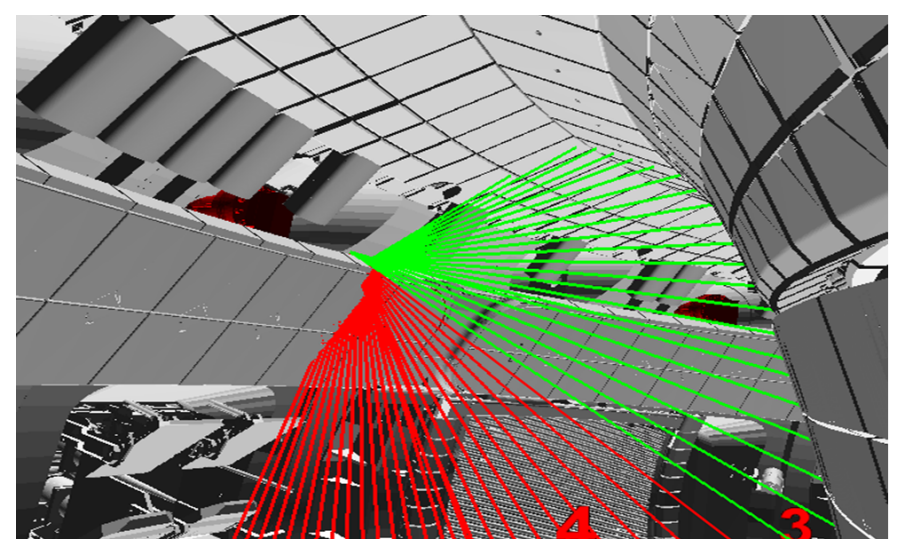

Figure 5: Lines of sight passing (green) and intersecting (green) with the housing of the planned AUG ELM control coils.

$3 \mathrm{D}$ profiles of the plasma, neutral particle and impurity parameters can be computed by the Edge Monte Carlo 3D (EMC3) - Eirene code package. This code is described in detail in $(4 ; 5 ; 6)$. Fig. 1 shows as an example the plasma density plotted into the same openGL space. With a few mouse clicks the user can load the LOS of a certain diagnostics (e.g. the Li-beam measuring the plasma density) and request the plasma parameters along this LOS. This 'virtual diagnostics' can then be compared the actual experimental diagnostics.

\section{Summary}

A generally available and easy-to-use software tool to visualise the complete 3D vessel structure of ASDEX Upgrade, diagnostic and magnetic geometry data and solutions of 3D plasma simulation codes was developed. By overplotting the $2 \mathrm{D}$ camera images an accurate calibration of the viewing geometry is achieved. This calibration allows for example an accurate and easy localisation of hot spots on plasma facing components, generation of input files for tomography algorithms and the reconstruction of dust or pellet trajectories. The tool furthermore can be used to compare the data of EMC3-Eirene simulations with experimental data and to test the compatibility of lines of sight with newly installed in vessel components.

[1] www.opengl.org

[2] J.C. Fuchs, et al., EPS 2002, ECA Vol.26B (2002) P-1.047

[3] J. Gafert, et al., EPS 2002, ECA Vol.26B (2002) P-1.123

[4] Y. Feng et al., Contrib. Plasma Phys. 44, No. 1-3, 57-69 (2004)

[5] M. Kobayashi, et al. Nucl. Fusion 47 61-73 (2007)

[6] Y. Feng, et al., EPS 2000, ECA Vol.24B (2000) 1188-1191 